\title{
The role of auxiliary states in state discrimination with linear optical devices.
}

\author{
Angelo Carollo ${ }^{1}$ and G.Massimo Palma ${ }^{1}$ \\ ${ }^{1}$ Dipartimento di Scienze Fisiche ed Astronomiche and INFM-Unità di Palermo, \\ Via Archirafi 36, I-90123 Palermo, Italy
}

(Dated: October 29, 2018)

\begin{abstract}
The role of auxiliary photons in the problem of identifying a state secretly chosen from a given set of L-photon states is analyzed. It is shown that auxiliary photons do not increase the ability to discriminate such states by means of a global measurement using only optical linear elements, conditional transformation and auxiliary photons.
\end{abstract}

PACS numbers: 03.67.Hk, 42.50.-p, 03.67.-a, 03.65.Bz

\section{INTRODUCTION}

Linear quantum optical devices have proved to be ideal system for the experimental implementation of several quantum information processing protocols like quantum cryptography [1], quantum teleportation [2] and quantum dense coding [3] to mention some. One of the main limitation of such systems is the difficulty to implement conditional dynamics as photons interfere but hardly interact with each other. However recently a proposal to implement probabilistic quantum computation 国 making use of linear optical devices has been put forward. In this proposal the difficulty to achieve experimentally conditional dynamics is circumvented by making use of auxiliary photons and obtaining a probability of success asymptotically close to one. On the other hand the problem of distinguishing completely photon states secretly chosen from a given set by means of linear devices has been addressed in several recent papers. In particular the set of Bell states has been considered in [5, [6] while the set of "non local without entanglement" if states has been analyzed in [8].

In this paper we will analyze the role of auxiliary photons in the problem of identifying the elements of an arbitrary set of orthogonal N-photon states by means of linear optical devices. We will show that auxiliary photons cannot increase our ability to identify which state has been chosen from a given input set.

The experimental setup we will consider is the one already discussed in [5, 8. Suppose that we want to discriminate exactly $L$-photon states over $M$ modes, randomly chosen from a known set of $K$ states. In our ideal setup the modes of the input states are mixed with an arbitrary number of auxiliary modes in a "black box" consisting of optical linear devices. The output modes of this box will be linked to the input ones by a unitary transformation $U$. It has been shown that any such unitary transformations of modes can be obtained by means of linear optical devices [9], like beam splitters and phase shifters. To ensure the largest possible generality in our measurement apparatus we will assume the possibility to perform conditional measurements. In practice this means what follows: assume that a measurement is made on one selected output mode while the others are kept in a delay loop and that, according to the outcome of the measurement, these modes are fed into a selected further black box, in a cascade setup ( see figure 11). The final assumption we will make is that our detectors have the ability to distinguish the number of incident photons. Although this assumption is unrealistic we have made it in order to guarantee the largest possible generality.

A first strategy to implement a measurement could be to mix the modes by means of linear devices and than perform, with the previously described cascade setup, conditional measurement on the output modes of such device. However, following [5, 8] we will adopt a more general strategy. We will assume to have at our disposal a set of as many additional modes as we like, here indicated with bosonic creation operators $c_{j}^{\dagger}$, with any number of photons we like and we will assume that these auxiliary modes can be mixed with modes $a_{i}^{\dagger}, b_{k}^{\dagger}$ in a black box.

In this scenario we will show that the use of auxiliary photons does not help in increasing the distinguishability of the input states. In other words we will demonstrate that given any two input $L$-photon states, they are completely distinguishable in the presence of auxiliary photons only if they are completely distinguishable in the absence of auxiliary photons. We will show that this is a consequence of the fact that, given a measurement outcome in the selected output mode, the error probability in presence of auxiliary photons has a direct linear relation with the error probability in absence of auxiliary photons. This assures that auxiliary photons cannot improve complete distinguishability.

The paper is structured as follows: in the next section we will describe our measurement setup and in section III we will prove our statement. As the mathematics involved is rather tedious to follow some of the details are discussed in appendix.

\section{STATEMENT OF THE PROBLEM}

As described already our measuring apparatus consists of a cascade of "black boxes", in which modes are linearly mixed, and partial measurements, which determine the sequence of unitary mixing. The first of such black box, denoted by $U_{1}$, is made out of linear optical elements and 


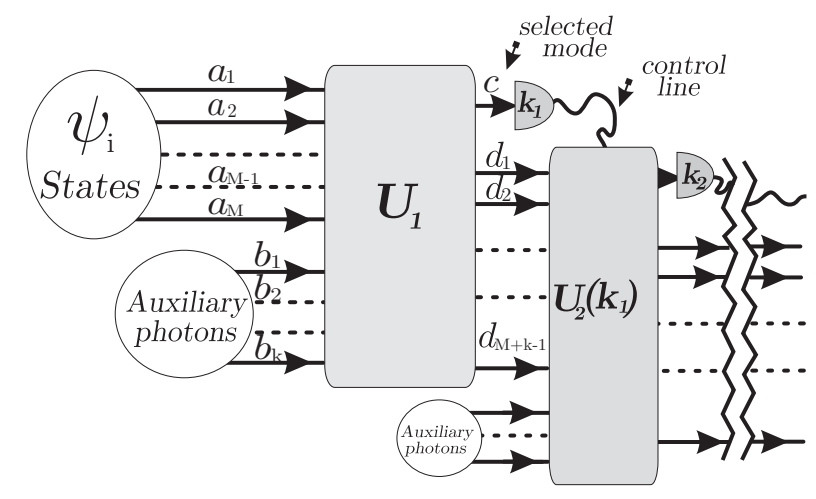

FIG. 1: Cascade setup in which the modes of the system states are mixed in a first "box" with auxiliary modes. Selected output mode is then measured and depending on its outcome the remaining output modes are fed in a new box. The process can be repeated over and over again

its input and output are a set of bosonic modes. The joint input modes consist of M "system" modes $\hat{a}_{i}^{\dagger}$ and an arbitrary number of auxiliary modes $\hat{b}_{i}^{\dagger}$. These input modes are unitarily mixed in the box into a set of output modes $\hat{c}^{\dagger}, \hat{d}_{i}^{\dagger}$ where the $\hat{c}^{\dagger}$ mode is the one on which a measurement will be performed. The measurement outcome determines the specific unitary mixing that will be performed in next step of the measurement, consisting of a second box $U_{2}$. While the measurement on mode $\hat{c}^{\dagger}$ is performed the photons in the remaining $\hat{d}_{i}^{\dagger}$ modes are kept in a waiting loop. The whole measurement procedure consists of a cascade of conditional measurements as described above.

The input state of the first block $U_{1}$ of the measuring apparatus is of the form $\left|\psi_{i}^{\text {tot }}\right\rangle=\left|\psi_{\text {aux }}\right\rangle \otimes\left|\psi_{i}\right\rangle$, where $\left|\psi_{i}\right\rangle$ is the state randomly chosen from a set of $K L$-photon states of $M$ modes we want to identify and $\left|\psi_{\text {aux }}\right\rangle$ is the state of the auxiliary modes. Such input state $\left|\psi_{i}^{\text {tot }}\right\rangle$ can be written as

$$
\left|\psi_{i}^{\text {tot }}\right\rangle=\left|\psi_{\text {aux }}\right\rangle \otimes\left|\psi_{i}\right\rangle=P_{\text {aux }}\left(\hat{b}_{k}^{\dagger}\right) P_{i}\left(\hat{a}_{n}^{\dagger}\right)|0\rangle
$$

where $P_{i}\left(\hat{a}^{\dagger}\right)$ is a polynomial of degree $N$ and $P_{a u x}\left(\hat{b}_{k}^{\dagger}\right)$ is a polynomial of arbitrary degree in the $\hat{b}_{k}^{\dagger}$

The corresponding output state is

$$
\left|\psi_{i}^{\text {tot }}\right\rangle=\tilde{P}_{a u x}\left(\hat{c}^{\dagger}, \hat{d}_{k}^{\dagger}\right) \tilde{P}_{\psi_{i}}\left(\hat{c}^{\dagger}, \hat{d}_{k}^{\dagger}\right)|0\rangle
$$

Where $\tilde{P}_{a u x}\left(\hat{c}^{\dagger}, \hat{d}_{k}^{\dagger}\right)$ and $\tilde{P}_{\psi_{i}}\left(\hat{c}^{\dagger}, \hat{d}_{k}^{\dagger}\right)$ are nothing but $P_{\text {aux }}\left(\hat{b}_{k}^{\dagger}\right)$ e $P_{i}\left(a_{k}^{\dagger}\right)|0\rangle$ written in terms of the creation and annihilation operators at the output of $U_{1}$.

We can expand $\tilde{P}_{a u x}, \tilde{P}_{\psi_{i}}$ in terms of decreasing powers of $\hat{c}^{\dagger}$ as follows

$$
\begin{aligned}
\tilde{P}_{a u x}\left(\hat{c}^{\dagger}, \hat{d}_{k}^{\dagger}\right) & =\sum_{n=0}^{n_{a}}\left(\hat{c}^{\dagger}\right)^{n} \widetilde{Q}_{a}^{(n)}\left(\hat{d}_{k}^{\dagger}\right) \\
\tilde{P}_{\psi_{i}}\left(\hat{c}^{\dagger}, \hat{d}_{k}^{\dagger}\right) & =\sum_{n=0}^{n_{s}}\left(\hat{c}^{\dagger}\right)^{n} \widetilde{Q}_{\psi_{i}}^{(n)}\left(\hat{d}_{k}^{\dagger}\right)
\end{aligned}
$$

In (4) $n_{s}$ is the largest order in $\hat{c}^{\dagger}$ for the $\tilde{P}_{\psi_{i}}$ with $i=1 . . K$ and by definition is independent on index $i$ $\left(\tilde{Q}_{\psi_{i}}\right.$ can be zero for some $i$ ). Analogously $n_{a}$ is defined as the order in $\hat{c}^{\dagger}$ of polynomial $\tilde{P}_{a u x}$. We can therefore rewrite (2) as

$$
\left|\psi_{i}^{t o t}\right\rangle=\sum_{n, m=0}^{n_{a}, n_{s}}\left(\hat{c}^{\dagger}\right)^{n+m} \widetilde{Q}_{a}^{(n)}\left(\hat{d}_{n}^{\dagger}\right) \widetilde{Q}_{\psi_{i}}^{(m)}\left(\hat{d}_{k}^{\dagger}\right)|0\rangle
$$

Let's suppose now that the number of photons on the selected mode $\hat{c}$ is measured. If $N$ is the outcome of such measurement the (unormalised) conditional state of the remaining modes can be we written as

$$
\left|\psi_{i}^{N}\right\rangle=\sum_{k} \widetilde{Q}_{a}^{(k)} \widetilde{Q}_{\psi_{i}}^{(N-k)}|0\rangle
$$

where $\max \left\{0, N-n_{s}\right\} \leq k \leq \min \left\{n_{a}, N\right\}$.

If the input states are to be distinguishable the conditional states $\left|\psi_{i}^{N}\right\rangle$ must be orthogonal for each possible value of $N$, i.e.

$$
\left\langle\psi_{i}^{N} \mid \psi_{j}^{N}\right\rangle=0 \quad \forall N, i \neq j
$$

Of particular interest in the following will be the case in which no photon is present in the auxiliary modes. In this case the distinguishability condition (7) becomes:

$$
\left\langle\psi_{i}^{N} \mid \psi_{j}^{N}\right\rangle_{n_{a}=0} \propto\left\langle 0\left|\widetilde{Q}_{\psi_{i}}^{(N) \dagger} \widetilde{Q}_{\psi_{j}}^{(N)}\right| 0\right\rangle=0 \quad \forall N, i \neq j
$$

where $\left|\psi_{i}^{N}\right\rangle_{n_{a}=0}$ is the conditional output state obtained from $\psi_{i}$ in the absence of auxiliary photons when $N$ photons are measured in mode $\hat{c}$. Here we have made of use of expression (6).

Out of the possible outcomes of the measurement of the number $N$ of photons in mode $\hat{c}$ we will concentrate on some particular outcomes, namely those for which

$$
n_{a} \leq N \leq n_{a}+n_{s}
$$

The reason of this particular choice will be shortly evident. Now, we define the following $n_{s}+1$-dimensional vectors:

$$
\mathbb{V}=\left(\begin{array}{c}
\left\langle\psi_{i}^{n_{a}+n_{s}} \mid \psi_{j}^{n_{a}+n_{s}}\right\rangle \\
\vdots \\
\left\langle\psi_{i}^{n_{a}+1} \mid \psi_{j}^{n_{a}+1}\right\rangle \\
\left\langle\psi_{i}^{n_{a}} \mid \psi_{j}^{n_{a}}\right\rangle
\end{array}\right) \quad \mathbb{U}=\left(\begin{array}{c}
\left\langle\psi_{i}^{n_{s}} \mid \psi_{j}^{n_{s}}\right\rangle_{n_{a}=0} \\
\vdots \\
\left\langle\psi_{i}^{1} \mid \psi_{j}^{1}\right\rangle_{n_{a}=0} \\
\left\langle\psi_{i}^{0} \mid \psi_{j}^{0}\right\rangle_{n_{a}=0}
\end{array}\right)
$$


whose elements are scalar products of conditional states after the measurement of $n$ photons in mode $\hat{c}$, with and without the auxiliary photons respectively. Using this vectors, conditions (8) can be rephrased as:

$$
\mathbb{U}=\underline{\mathbf{0}}
$$

while a necessary condition for distinguishability with auxiliary photon can be expressed as (see (7)):

$$
\mathbb{V}=\underline{\mathbf{0}}
$$

The central point of this paper is that, as we will show, vectors $\mathbb{V}$ and $\mathbb{U}$ are linearly connected through a matrix whose determinant is not vanishing,i.e.

$$
\mathbb{V}=\mathbf{M U} \quad \operatorname{det}(\mathbf{M}) \neq 0
$$

The elements of this matrix only depend on the auxiliary photon states and are indedependent on the states of the system. This implies that conditions (11a) and (11b) are completely equivalent or, in other words, that distinguishability in absence of auxiliary photon is a necessary condition for distinguishability in presence of auxiliary photons.

\section{PROOF THAT AUXILIARY PHOTONS DO NOT INCREASE COMPLETE DISTIGUISHABILITY}

In order to demonstrate the previous statement we define the following $n_{s}+1$-dimensional vector:

$$
\mathbb{U}^{\prime} \equiv\left(\begin{array}{c}
\left\langle 0\left|\widetilde{Q}_{\psi_{i}}^{\left(n_{s}\right) \dagger} \widetilde{Q}_{\psi_{j}}^{\left(n_{s}\right)}\right| 0\right\rangle \\
\vdots \\
\left\langle 0\left|\widetilde{Q}_{\psi_{i}}^{(1) \dagger} \widetilde{Q}_{\psi_{j}}^{(1)}\right| 0\right\rangle \\
\left\langle 0\left|\widetilde{Q}_{\psi_{i}}^{(0) \dagger} \widetilde{Q}_{\psi_{j}}^{(0)}\right| 0\right\rangle
\end{array}\right)
$$

From eq. (8) immediately follows that $\mathbb{U}^{\prime}$ is linearly connected with $\mathbb{U}$.

In this section we will demonstrate the following

Theorem Given the vectors $\mathbb{V}$ and $\mathbb{U}^{\prime}$, defined in eq (10) and eq. (13) respectively, it follows that:

$$
\mathbb{V}=\mathbf{M}^{\prime} \mathbb{U}^{\prime}
$$

where $\mathbf{M}^{\prime}$ is a triangular matrix of this form:

$$
\mathbf{M}^{\prime}=\left(\begin{array}{ccccc}
\mathcal{D} & 0 & \cdots & 0 & 0 \\
m_{10} & \mathcal{D} & \cdots & 0 & 0 \\
m_{20} & m_{21} & \ddots & 0 & 0 \\
\vdots & \vdots & & \vdots & \vdots \\
m_{n_{s} 0} & m_{n_{s} 1} & \cdots & m_{n_{s} n_{s}-1} & \mathcal{D}
\end{array}\right)
$$

with

$$
\mathcal{D} \equiv\left\langle 0\left|\widetilde{Q}_{a}^{\left(n_{a}\right) \dagger} \widetilde{Q}_{a}^{\left(n_{a}\right)}\right| 0\right\rangle
$$

Remark By definition $\mathcal{D}=\| \widetilde{Q}_{a}^{\left(n_{a}\right)}\left(\hat{d}_{k}^{\dagger}\right)|0\rangle \|^{2}>0$, being $\widetilde{Q}_{a}^{\left(n_{a}\right)}$ the first non vanishing term of the expansion (3). From this follows that

$$
\operatorname{det}\left(\mathbf{M}^{\prime}\right)=\mathcal{D}^{n_{s}+1}>0 \text {. }
$$

which implies eq. (19).

Before entering into the details of our proof we briefly introduce some notation.

From (6) follows that the scalar product between the (unormalised) states $\left|\psi_{i}^{N}\right\rangle,\left|\psi_{j}^{N}\right\rangle$ obtained after the measurement of $N$ photons in mode $\hat{c}$ is

$$
\left\langle\psi_{i}^{N} \mid \psi_{j}^{N}\right\rangle=\sum_{n, m}\left\langle 0\left|\widetilde{Q}_{\psi_{i}}^{(N-m) \dagger} \widetilde{Q}_{a}^{(m) \dagger} \widetilde{Q}_{a}^{(n)} \widetilde{Q}_{\psi_{i}}^{(N-n)}\right| 0\right\rangle
$$

with $\max \left\{0, N-n_{s}\right\} \leq n, m \leq \min \left\{n_{a}, N\right\}$.

Let's define $N_{m}=n_{a}+n_{s}$ and express the eq. (18) in an alternative form:

$$
\left\langle\psi_{i}^{N_{m}-s} \mid \psi_{j}^{N_{m}-s}\right\rangle=\sum_{n, m} \mathcal{C}_{m, n}^{(s)}(i, j)
$$

where $s$ is defined as: $s \equiv N_{m}-N$, and obviously $0 \leq$ $s \leq N_{m}$, while $\mathcal{C}_{n, m}^{(s)}$ is defined as:

$$
\begin{aligned}
& \mathcal{C}_{n, m}^{(s)}(i, j) \equiv \\
& \equiv\left\langle 0\left|\widetilde{Q}_{\psi_{i}}^{\left(n_{s}-n\right) \dagger} \widetilde{Q}_{a}^{\left(n_{a}-s+n\right) \dagger} \widetilde{Q}_{a}^{\left(n_{a}-s+m\right)} \widetilde{Q}_{\psi_{j}}^{\left(n_{s}-m\right)}\right| 0\right\rangle
\end{aligned}
$$

In this section we will make use of the following properties of coefficients $\mathcal{C}_{n, m}^{(s)}(i, j)$, which are demonstrated in appendix A:

\section{Property 1 Symmetry:}

$$
\mathcal{C}_{n, m}^{(s)}(i, j)=\mathcal{C}_{m, n}^{(s)}(i, j)
$$

Property 2 Recurrence relation:

$$
\begin{gathered}
\mathcal{C}_{m, n}^{(s)}(i, j)= \\
\delta_{n, m}\left\langle 0\left|\widetilde{Q}_{\psi_{i}}^{\left(n_{s}-n\right) \dagger} \widetilde{Q}_{\psi_{j}}^{\left(n_{s}-n\right)}\right| 0\right\rangle\left\langle 0\left|\widetilde{Q}_{a}^{\left(n_{a}-s+n\right) \dagger} \widetilde{Q}_{a}^{\left(n_{a}-s+n\right)}\right| 0\right\rangle- \\
-\sum_{k}^{k}\left(\begin{array}{c}
n_{a}-s+m+k \\
k
\end{array}\right)\left(\begin{array}{c}
n_{s}-n+k \\
k
\end{array}\right) \mathcal{C}_{n-k, m}^{(s-k)}(i, j)
\end{gathered}
$$

with $n \geq m, 1 \leq k \leq \min \{n, s-m\}$, and where $\delta_{n, m}$ is the Kroneker symbol. 
It is straightforward to verify by recursion that Property 2 implies the following expression for the coefficients $\mathcal{C}_{m, n}^{(s)}(i, j)$ :

$$
\mathcal{C}_{m, n}^{(s)}(i, j)=\sum_{p} \mathcal{A}_{p}^{(s)}(n, m)\left\langle 0\left|\widetilde{Q}_{\psi_{i}}^{\left(n_{s}-p\right) \dagger} \widetilde{Q}_{\psi_{j}}^{\left(n_{s}-p\right)}\right| 0\right\rangle
$$

where $\min \{n, m\} \leq p \leq \max \{0, n+m-s\}$, and:

$$
\begin{aligned}
& \mathcal{A}_{n}^{(s)}(n, n)=\left\langle 0\left|\widetilde{Q}_{a}^{\left(n_{a}-s+n\right) \dagger} \widetilde{Q}_{a}^{\left(n_{a}-s+n\right)}\right| 0\right\rangle \\
& \mathcal{A}_{p}^{(s)}(n, m)= \\
& -\sum_{k} k !\left(\begin{array}{c}
n_{a}-s+m+k \\
k
\end{array}\right)\left(\begin{array}{c}
n_{s}-n+k \\
k
\end{array}\right) \mathcal{A}_{p}^{(s-k)}(n-k, m)
\end{aligned}
$$

where the eq. 22d is valid for $n \geq m$, and $1 \leq k \leq$ $\min \{n-p, s-m\}$.

The above expression gives us a recursive method for calculating all the coefficient of expression (19) once the auxiliary photons states are known. With the help of eqs. (22) it is possible to derive the following properties of coefficients $\mathcal{A}_{p}^{(s)}(n, m)$,

- $\mathcal{A}$ 's only depend on the auxiliary states and do not depend at all on the states $\psi_{i}$ and $\psi_{j}$.

- $\mathcal{A}$ 's are real numbers.

- $\mathcal{A}$ 's are symmetric in respect to the indices $\mathrm{n}$ and m.

It is now possible to cast equation (19) as a sum whose elements factor in a product of coefficients depending only on system states with coefficients depending only on auxiliary states. Let's consider eq. (19) in the case of $s \leq n_{s}$ :

$$
\begin{aligned}
\left\langle\psi_{i}^{N_{m}-s} \mid \psi_{j}^{N_{m}-s}\right\rangle= & \sum_{n, m=\max \left\{0, s-n_{a}\right\}}^{s} \mathcal{C}_{m, n}^{(s)}(i, j) \\
= & \mathcal{C}_{s, s}^{(s)}(i, j)+\sum_{n, m}^{\prime} \mathcal{C}_{m, n}^{(s)}(i, j)
\end{aligned}
$$

where the sum $\sum_{n, m}^{\prime}$ is extended over $\max \left\{0, s-n_{a}\right\} \leq n, m \leq s \quad$ with $\min \{n, m\}<s$. From eqs. (22) follows that

$$
\mathcal{C}_{s, s}^{(s)}(i, j)=\mathcal{D} \cdot\left\langle 0\left|\widetilde{Q}_{\psi_{i}}^{\left(n_{s}-s\right) \dagger} \widetilde{Q}_{\psi_{j}}^{\left(n_{s}-s\right)}\right| 0\right\rangle
$$

where $\mathcal{D}$, the coefficient previously defined as

$$
\mathcal{D}=\left\langle 0\left|\widetilde{Q}_{a}^{\left(n_{a}\right) \dagger} \widetilde{Q}_{a}^{\left(n_{a}\right)}\right| 0\right\rangle=\mathcal{A}_{s}^{(s)}(s, s)
$$

is by definition independent from $s$; while in the second term of (23)

$$
\mathcal{C}_{m, n}^{(s)}(i, j)=\sum_{p} \mathcal{A}_{p}^{(s)}(n, m)\left\langle 0\left|\widetilde{Q}_{\psi_{i}}^{\left(n_{s}-p\right) \dagger} \widetilde{Q}_{\psi_{j}}^{\left(n_{s}-p\right)}\right| 0\right\rangle
$$

where $\max \{n+m-s\} \leq p \leq \min \{n, m\}<s$. Finally with the help of the eqs. 24, 25, 26) we can write:

$$
\begin{aligned}
\left\langle\psi_{i}^{N_{m}-s} \mid \psi_{j}^{N_{m}-s}\right\rangle & =\mathcal{D} \cdot\left\langle 0\left|\widetilde{Q}_{\psi_{i}}^{\left(n_{s}-s\right) \dagger} \widetilde{Q}_{\psi_{j}}^{\left(n_{s}-s\right)}\right| 0\right\rangle+ \\
+ & \sum_{p} \mathcal{B}_{p}^{(s)} \cdot\left\langle 0\left|\widetilde{Q}_{\psi_{i}}^{\left(n_{s}-p\right) \dagger} \widetilde{Q}_{\psi_{j}}^{\left(n_{s}-p\right)}\right| 0\right\rangle
\end{aligned}
$$

with $\max \left\{0, s-2 n_{a}\right\} \leq p \leq s-1$ and

$$
\mathcal{B}_{p}^{(s)}=\sum_{n, m} \mathcal{A}_{p}^{(s)}(n, m)
$$

This expression allows us to write:

$$
\mathbb{V}=\mathbf{M}^{\prime} \mathbb{U}^{\prime}
$$

where

$$
\mathbf{M}^{\prime}=\left(\begin{array}{ccccc}
\mathcal{D} & 0 & \cdots & 0 & 0 \\
\mathcal{B}_{0}^{(1)} & \mathcal{D} & \cdots & 0 & 0 \\
\mathcal{B}_{0}^{(2)} & \mathcal{B}_{1}^{(2)} & \cdots & 0 & 0 \\
\vdots & \vdots & \ddots & \vdots & \vdots \\
\mathcal{B}_{0}^{\left(n_{s}-1\right)} & \mathcal{B}_{1}^{\left(n_{s}-1\right)} & \ldots & \mathcal{D} & 0 \\
\mathcal{B}_{0}^{\left(n_{s}\right)} & \mathcal{B}_{1}^{\left(n_{s}\right)} & \cdots & \mathcal{B}_{n_{s}-1}^{\left(n_{s}\right)} & \mathcal{D}
\end{array}\right)
$$

whose determinant is

$$
\operatorname{det}\left(\mathbf{M}^{\prime}\right)=\mathcal{D}^{n_{s}+1}>0 \text {. }
$$

This completes our proof.

\section{CONCLUSIONS}

In this manuscript we have discussed the role of auxiliary photons in state discrimination with linear optical devices. The cascade setup we have considered is of large generality. We have shown that for such setup auxiliary photons do not increase complete distinguishibility. This results wants to be a contribution to the assessment of the role of resources in quantum information processing with linear optical devices.

\section{Acknowledgments}

We would like to thank J.Calsamiglia, N.Lütkenhaus, C.Simon and A.Zeilinger for helpful discussions. This work was supported in part by the EU under grants TMR - ERB FMR XCT 96-0087 - "The Physics of Quantum Information" IST - 1999 - 11053 - EQUIP,"Entanglement in Quantum Information Processing and Communication". 


\section{APPENDIX A}

In this section we will prove Property 1 and Property 2 of section [II. To this goal we will make use of the following lemma:

Lemma If $\left[\tilde{P}_{a u x} \tilde{P}_{\psi_{i}}\right]=0$, where $\tilde{P}_{a u x}$ and $\tilde{P}_{\psi_{i}}$ are defined by (2), then:

$$
\widetilde{Q}_{a}^{(n)} \widetilde{Q}_{\psi_{i}}^{(m) \dagger}=\sum_{k} k !\left(\begin{array}{c}
m+k \\
k
\end{array}\right)\left(\begin{array}{c}
n+k \\
k
\end{array}\right) \widetilde{Q}_{\psi_{i}}^{(m+k) \dagger} \widetilde{Q}_{a}^{(n+k)}
$$

where $0 \leq k \leq \min \left\{n_{s}-m, n_{a}-n\right\}$.

Proof. From eqs. (3) and (4) follows:

$$
\begin{aligned}
& {\left[\tilde{P}_{a u x} \tilde{P}_{\psi_{i}}\right]=} \\
& =\sum_{n=0}^{n_{a}} \sum_{m=0}^{n_{s}}\left\{\hat{c}^{\dagger n} \hat{c}^{m} \widetilde{Q}_{a}^{(n)} \widetilde{Q}_{\psi_{i}}^{(m) \dagger}-\hat{c}^{m} \hat{c}^{\dagger n} \widetilde{Q}_{\psi_{i}}^{(m) \dagger} \widetilde{Q}_{a}^{(n)}\right\}=0
\end{aligned}
$$

Furthermore the following property for creation and annihilation operators holds:

$$
\hat{c}^{m} \hat{c}^{\dagger n}=\sum_{k=0}^{\min \{n, m\}} k !\left(\begin{array}{l}
m \\
k
\end{array}\right)\left(\begin{array}{l}
n \\
k
\end{array}\right) \hat{c}^{\dagger n-k} \hat{c}^{m-k}
$$

Inserting eq. A3 in eq. A2 and reordering elements in the sum it can be shown that

$$
\left[\tilde{P}_{a u x} \tilde{P}_{\psi_{i}}\right]=\sum_{n=0}^{n_{a}} \sum_{m=0}^{n_{s}} \hat{f}_{n, m}\left(\hat{d}_{k}^{\dagger}, \hat{d}_{k}^{m}\right) \hat{c}^{m} \hat{c}^{\dagger n}=0
$$

where

$$
\begin{aligned}
& \hat{f}_{n, m}=\left\{\widetilde{Q}_{a}^{(n)} \widetilde{Q}_{\psi_{i}}^{(m) \dagger}-\right. \\
& \left.-\sum_{k} k !\left(\begin{array}{c}
m+k \\
k
\end{array}\right)\left(\begin{array}{c}
n+k \\
k
\end{array}\right) \widetilde{Q}_{\psi_{i}}^{(m+k) \dagger} \widetilde{Q}_{a}^{(n+k)}\right\}=0
\end{aligned}
$$

in which $0 \leq k \leq \min \left\{n_{s}-m, n_{a}-n\right\}$.

Proof of Property 2.

Eq. (A1) can be rewritten in the following form:

$$
\begin{aligned}
\widetilde{Q}_{\psi_{i}}^{(m) \dagger} \widetilde{Q}_{a}^{(n)}=\widetilde{Q}_{a}^{(n)} \widetilde{Q}_{\psi_{i}}^{(m) \dagger}- \\
\quad-\sum_{k} k !\left(\begin{array}{c}
m+k \\
k
\end{array}\right)\left(\begin{array}{c}
n+k \\
k
\end{array}\right) \widetilde{Q}_{\psi_{i}}^{(m+k) \dagger} \widetilde{Q}_{a}^{(n+k)}
\end{aligned}
$$

where $1 \leq k \leq \min \left\{n_{s}-m, n_{a}-n\right\}$. Inserting eq. (A6) in the definition (20) it can be shown that:

$$
\begin{aligned}
& \mathcal{C}_{n, m}^{(s)}(i, j)= \\
& =\left\langle 0\left|\widetilde{Q}_{a}^{\left(n_{a}-s+n\right) \dagger} \widetilde{Q}_{a}^{\left(n_{a}-s+n\right)} \widetilde{Q}_{\psi_{i}}^{\left(n_{s}-n\right) \dagger} \widetilde{Q}_{\psi_{j}}^{\left(n_{s}-n\right)}\right| 0\right\rangle- \\
& -\sum_{k} k !\left(\begin{array}{c}
n_{a}-s+m+k \\
k
\end{array}\right)\left(\begin{array}{c}
n_{s}-n+k \\
k
\end{array}\right) \mathcal{C}_{n-k, m}^{(s-k)}(i, j)
\end{aligned}
$$

with $1 \leq k \leq \min \{n, s-m\}$. As all the states $\psi_{i}$ contain a fixed number $L$ of photons $\tilde{P}_{\psi_{i}}\left(c^{\dagger}, d_{k}^{\dagger}\right)$ is a homogeneous polynomial of degree $L$ in $c^{\dagger}$ and $d_{k}^{\dagger}$ and therefore the generic $\widetilde{Q}_{\psi_{i}}^{(n)}$ is a homogeneous polynomial of degree $L-n$ in $d_{k}^{\dagger}$. As a consequence $\widetilde{Q}_{\psi_{i}}^{\left(n_{s}-n\right) \dagger} \widetilde{Q}_{\psi_{j}}^{\left(n_{s}-m\right)}|0\rangle=0$ unless $n \leq m$. From this follows that for $n \geq m$ the first term on the right hand side of eq. (A7) can be expressed as:

$\delta_{n, m}\left\langle 0\left|\widetilde{Q}_{\psi_{i}}^{\left(n_{s}-n\right) \dagger} \widetilde{Q}_{\psi_{j}}^{\left(n_{s}-n\right)}\right| 0\right\rangle\left\langle 0\left|\widetilde{Q}_{a}^{\left(n_{a}-s+n\right) \dagger} \widetilde{Q}_{a}^{\left(n_{a}-s+n\right)}\right| 0\right\rangle$

Above we have introduced the completeness relation $\sum_{\{\mathbf{n}\}}|\mathbf{n}\rangle\langle\mathbf{n}|$, where $|\mathbf{n}\rangle$ is a Fock states of the relevant modes. Note that only the term corresponding to $|0\rangle\langle 0|$ survives. Hence the proof is complete.

\section{Proof of Property1.}

By definition:

$$
\begin{aligned}
\mathcal{C}_{m, n}(i, j) & =\left\langle 0\left|\widetilde{Q}_{\psi_{i}}^{\left(n_{s}-m\right) \dagger} \widetilde{Q}_{a}^{\left(n_{a}-n\right) \dagger} \widetilde{Q}_{a}^{\left(n_{a}-m\right)} \widetilde{Q}_{\psi_{j}}^{\left(n_{s}-n\right)}\right| 0\right\rangle \\
& =\left(\left\langle 0\left|\widetilde{Q}_{\psi_{j}}^{\left(n_{s}-n\right) \dagger} \widetilde{Q}_{a}^{\left(n_{a}-m\right) \dagger} \widetilde{Q}_{a}^{\left(n_{a}-n\right)} \widetilde{Q}_{\psi_{i}}^{\left(n_{s}-m\right)}\right| 0\right\rangle\right)^{*} \\
& =\mathcal{C}_{n, m}^{*}(j, i)
\end{aligned}
$$

from the above equation and eq.(21) immediately follows that for $m<n$

$$
\begin{aligned}
& \mathcal{C}_{m, n}^{(s)}(i, j)= \\
& -\sum_{k=1}^{E} k !\left(\begin{array}{c}
n_{a}-s+m+k \\
k
\end{array}\right)\left(\begin{array}{c}
n_{s}-n+k \\
k
\end{array}\right) \mathcal{C}_{m, n-k}^{(s-k)}(i, j)
\end{aligned}
$$

As $\mathcal{C}_{0,0}^{(0)}(i, j)$ is trivially symmetric, it follows from recursion on $s$, that all $\mathcal{C}_{n, m}^{(s)}(i, j)$ are symmetric under exchange of indices $n$ and $m$.
[1] T. Jennewein, C. Simon, G. Weihs, H. Weinfurter, and A. Zeilinger, Phys. Rev. Lett. 84, 4729 (2000)
[2] D. Bouwmeester, J.-W. Pan, K. Mattle, M. Eibl, H. We- 
infurter and A. Zeilinger, Nature 390, 575 (1997).

[3] K. Mattle, H. Weinfurter, P.G. Kwiat and A. Zeilinger, Phys. Rev. Lett. 76, 4656 (1997).

[4] E. Knill, R. Laflamme and G.J. Milburn, Nature 409, 49 (2001).

[5] N. Lütkenhaus, J. Calsamiglia, K.-A. Suominen, Phys. Rev. A 59 , 3295 (1999).

[6] J.Calsamiglia and N.Lütkenhaus, App. Phys. B, 67-71 (2001).
[7] C.H. Bennett, D. DiVincenzo, C.A. Fuchs, T. Mor, E. Rain, P.W. Shor, J.A. Smolin, W.K. Wootters, Phys. Rev. A 59, 1070 (1999).

[8] A. Carollo, G.M. Palma, C. Simon and A. Zeilinger eprint:quant-ph/0102124 Phys. Rev. A (in press)

[9] M.Reck, A.Zeilinger,H.J.Bernstein and P.Bertani Phys.Rev.Lett. 73, 58 (1994). 